\title{
Efetividade de Curso de Capacitação em Medida da Altura Uterina para enfermeiros e graduandos de Enfermagem
}

\author{
Effectiveness of a Training Course on the Measure of Uterine Height for nurses and nursing students \\ Efectividad de Curso de Capacitación en Medida de la Altura Uterina para enfermeros \\ y estudiantes de Enfermería
}

Submissão: 08-09-2011 Aprovação: 26-10-2012

Camila Caroline de Amorim Paiva', Djacyr Magna Cabral Freire"

' Universidade Federal da Paraíba, Centro de Ciências Médicas, Curso de Medicina, Graduanda. João Pessoa-PB, Brasil. " Universidade Federal da Paraíba, Centro de Ciências da Saúde, Escola Técnica de Saúde. João Pessoa-PB, Brasil.

\section{RESUMO}

Estudo longitudinal e prospectivo que procurou quantificar a variabilidade intra e interobservadores da medida da altura uterina (AU) e verificar a contribuição de um curso capacitatório para enfermeiros e graduandos de Enfermagem no viés de aferição da medida. Foram selecionados dois participantes, que realizaram duas medidas em vinte gestantes antes e após o curso. Calculou-se a variabilidade intra e interobservadores, o coeficiente de correlação intraclasse, o erro técnico de medida absoluto e relativo e a fiabilidade da medida. O teste $t$ pareado mostrou redução estatisticamente significante $(p=0,0396)$ no viés de aferição. Conclui-se que a capacitação de enfermeiros e graduandos de Enfermagem na técnica de medição da AU reduz significativamente a variabilidade intraobservador e interobservadores.

Descritores: Assistência Pré-Natal; Precisão; Ensino; Enfermagem; Graduação.

\section{ABSTRACT}

A prospective, longitudinal study sought to quantify the intra and inter-observer variability of the uterine height (UH) measurement and verify the contribution of a training course for nurses and nursing students in measurement bias. We selected two participants who performed two measurements in 20 pregnant women before and after the course. We calculated the intra and inter-observer variability, the coefficient of intraclass correlation, the absolute and relative technical error of measurement and the measure reliability. The paired $t$ test showed a statistically significant reduce $(p=0.0396)$ in the measurement bias. We conclude that the training of nurses and nursing students in the technical measurement of UH significantly reduces intra and inter-observer variability.

Key words: Prenatal Care; Precision; Teaching; Nursing; Undergraduate.

\section{RESUMEN}

Estudio prospectivo, longitudinal tratado de cuantificar la variabilidad intra y inter-observador de medición de el altura uterina (AU) y verificar la contribución de un curso capacitación para enfermeras y estudiantes de enfermería en los sesgos de medición. Fueran seleccionados dos participantes que realizaron dos mediciones de cada 20 mujeres embarazadas antes y después del curso. Se calculó la variabilidad intra y inter-observador, el coeficiente de correlación intraclase, el error técnico de medición absoluta y relativa y la fiabilidad de la medida. El teste $t$ de Student mostró reducción estadísticamente significativa $(p=0,0396)$ en el sesgo. Se concluye que la formación de enfermeras y estudiantes de enfermería en la técnica de medición de la AU redujo significativamente la variabilidad intra y inter-observador.

Palabras clave: Atención Prenatal; Precisión; Enseñanza; Enfermería; Pregrado. 


\section{INTRODUÇÃO}

O papel da assistência pré-natal em termos de prevenção das complicações maternas e fetais é amplamente reconhecido, principalmente em países onde a saúde pública é deficiente. Um importante determinante do impacto dos serviços de assistência pré-natal em uma população é a qualidade destes serviços. Com relação à qualidade da atenção dedicada ao pré-natal, os principais problemas apontados na literatura referem-se à falta de cumprimento das normas e rotinas padronizados pelo Ministério da Saúde (MS), à falta de preenchimento de registros e à constatação de que os cuidados prestados não são direcionados às reais necessidades da gestante ${ }^{(1)}$.

Através da área técnica da assistência integral à saúde da mulher, o MS lançou em 2000 um novo programa de normas e rotinas da assistência pré-natal com o principal objetivo de melhorar a qualidade desta assistência. Uma das principais preocupações que o MS teve na confecção deste manual técnico foi a de sensibilizar os profissionais de saúde para a necessidade de reduzir os altos índices de mortalidade materna e perinatal no Brasil, demonstrando que a adesão das mulheres ao pré-natal esta relacionada com a qualidade da assistência prestada ao serviço.

Para implementar as atividades de normatização do controle pré-natal, é necessário dispor de um instrumento que permita identificá-las no contexto amplo de suas vidas e mapear os riscos a que cada uma delas está exposta. Isso permitirá a orientação e encaminhamentos adequados em cada momento da gravidez. O retardo de crescimento fetal, desvio quanto ao crescimento uterino, números de fetos e volume do líquido amniótico estão incluídos no quadro de fatores de risco na gravidez. A presença, ou não, destes fatores de risco é identificada pelo médico ou enfermeira, através da medida da altura uterina no momento da consulta, permitindo orientar oportunamente as condutas adequadas a serem tomadas.

A medida da $\mathrm{AU}$ foi selecionada para ser amplamente discutida neste estudo por ser um dos procedimentos de baixo custo, de fácil manuseio, excelente método de triagem para possíveis diagnósticos de desvios do crescimento fetal ou do líquido amniótico; porém, observamos que é pouco utilizada pelos profissionais nos registros da gestante. Para que esta técnica seja realizada com melhor precisão, vários critérios devem ser levados em consideração: a variabilidade interobservador e intraobservador, a padronização da técnica, a habilidade em desenvolver o procedimento, entre outros. Portanto, o presente estudo pretende reportar-se na literatura e conceituar o que existe de mais novo em relação à medida da AU como método de screening.

Historicamente, a medida da AU começou a ser utilizada na prática obstétrica como um procedimento subjetivo, utilizando-se a técnica de comparação da altura do fundo uterino com pontos anatômicos de referência no abdômen materno. Diversos estudos posteriores deram suporte à observação de que os referenciais anatômicos no abdômen materno apresentam uma variabilidade biológica grande demais para permitir uma avaliação acurada do crescimento uterino durante a gestação $^{(2)}$. Além da variabilidade biológica, Engstrom et al. ${ }^{(3)}$ enumeraram outros três fatores que parecem contribuir para a baixa confiabilidade dessa técnica: a) incapacidade para avaliar adequadamente o crescimento uterino quando a unidade de medida da altura uterina utilizada é "dedos de largura" ou "uma fração da distância entre dois referenciais anatômicos", ao invés da unidade de medida "centímetro"; b) discordância entre obstetras e pesquisadores sobre a relação precisa entre o fundo uterino e os referenciais anatômicos em cada estágio da gestação; c) variabilidade entre as mulheres no momento em que o fundo uterino alcança cada referencial.

Frequentemente, nas consultas pré-natais, as medidas da $\mathrm{AU}$ são usadas por médicos e enfermeiros para verificar se o crescimento fetal está progredindo normalmente ${ }^{(3)}$. São também usadas para identificar erros na determinação da idade gestacional em semanas. A falta de um padrão de referência é um problema para estes profissionais porque várias técnicas para medir a AU têm sido descritas na literatura. A eficácia destas várias técnicas de medidas não tem sido estudada e comparada adequadamente. Neste contexto é importante que a equipe de saúde esteja treinada com a padronização de uma única técnica para medição da $\mathrm{AU}$ e, desse modo, reduzir a níveis aceitáveis o viés de aferição que resulta de técnica inadequada.

Consideramos importante quantificar a variabilidade intra e interobservadores da medida da $\mathrm{AU}$, identificar os fatores que interferem na confiabilidade dessas medidas e verificar se um programa de treinamento com desenvolvimento de habilidades específicas contribui para obter resultados confiáveis e para uma maior efetividade na detecção de desvios do crescimento fetal.

A medida da altura uterina (AU) é uma técnica simples para avaliar o crescimento fetal durante as consultas do pré-natal e a utilização de uma curva padrão de crescimento da $\mathrm{AU}$ em função da idade gestacional é eficaz para diagnosticar retardo de crescimento intrauterino (RCIU). Se for utilizada com os objetivos semelhantes, a ultra-sonografia parece oferecer maior precisão, no entanto é um método mais sofisticado, de maior custo e que necessita de profissionais especializados.

A medida da AU é um importante procedimento auxiliar no diagnóstico clínico, não só utilizada para rastreamento dos desvios de crescimento intra-uterino, como também para identificar outras complicações na gravidez, tais como gestações múltiplas, desordem do líquido amniótico e casos de mola hidatiforme. As medidas da AU também podem ser usadas na identificação de erros na estimativa da idade da gestação em semanas, baseada na data da última menstruação (DUM). Com este procedimento, se estima o baixo peso neonatal em um centro de atenção primária, com baixos custos, podendo referir as gestantes de risco para que sejam assistidas em um nível de complexidade que o caso requeira ${ }^{(2)}$. O MS também considera que a medida da AU é uma técnica simples que pode ser usada no serviço de pré-natal, em nível de atenção primária, para predizer o baixo peso ao nascer.

A demanda por tecnologia sofisticada poderia ser reduzida se houvesse um adequado monitoramento da mulher durante o período gestacional ${ }^{(5)}$. Com este mesmo fim, a vigilância do crescimento fetal pode ser efetuada através da mensuração seriada da AU com uma fita métrica. 
Além disso, com a identificação cada vez mais frequente de gestações de alto-risco, o conhecimento da idade gestacional torna-se indispensável nas decisões clínicas, tanto para intervenções diagnósticas como terapêuticas. Para isso, novos instrumentos têm determinado com grandes vantagens a idade gestacional, como, por exemplo, a ultra-sonografia. Entretanto, são métodos tecnicamente mais complexos, nem sempre disponíveis em todas as comunidades e, nesses casos, a medição da $\mathrm{AU}$ em intervalos regulares é capaz de fornecer a idade gestacional com excelente precisão ${ }^{(6)}$.

$\mathrm{Na}$ rotina, observamos que há profissionais que não realizam a medida da altura uterina e, daqueles que a fazem, existem os que não registram o resultado na curva de $\mathrm{AU}$ e não o interpretam. Estudos realizados em 18 áreas de nove dos 36 municípios mais pobres das regiões Norte e Nordeste do Brasil mostram as seguintes porcentagens da realização da medida de $\mathrm{AU}$ em gestantes, durante o pré-natal, nos anos 2002 e 2005: 54\% e 77\%, respectivamente ${ }^{(7)}$.

Por outro lado, em uma avaliação transversal realizada no município do Rio Grande, no estado do Rio Grande do Sul (Brasil), mostrou-se que, entre as 230 puérperas incluídas na amostra, $228(99,1 \%)$ haviam realizado pelos menos um exame ultrassonográfico durante a assistência pré-natal ${ }^{(8)}$. Esse estudo ainda apresenta dados como a relação do número de exames ultrassonográficos realizados e o tipo de assistência pré-natal, obtendo os seguintes resultados: as pacientes do grupo dos convênios ou particulares foram mais frequentemente submetidas a quatro ou mais exames; o grupo de gestantes que realizou o pré-natal nos postos de saúde do município (SUS) foi o mais se submetido a dois exames de ultrassonografia $(54,4 \%)$; as pacientes que realizaram o pré-natal no Hospital Universitário da Fundação Universidade do Rio Grande (FURG) foram submetidas, na maioria dos casos, a um exame ecográfico (30,6\%).

Para aqueles que preenchem e interpretam a curva de $\mathrm{AU}$ na assistência pré-natal, torna-se importante avaliar qual gráfico de curva de crescimento da AU para a IG eles utilizam, já que cada população possui características peculiares que podem gerar resultados falso-positivos e falso-negativos se utilizados em uma população diferente. O gráfico recomendado pelo MS do Brasil foi elaborado a partir de dados do Centro Latino-Americano de Perinatologia (CLAP). Entretanto, há autores ${ }^{(9)}$ que têm demonstrado discrepância entre os valores de curvas de $\mathrm{AU}$ apresentadas na literatura quando aplicadas a populações diferentes das que forneceram os parâmetros para sua construção ${ }^{(10-17)}$. As diferenças observadas foram explicadas não apenas pelo caráter regional das diferentes curvas, mas também por fatores como a técnica de medição da AU.

Apoiados na experiência prática da consulta de enfermagem à gestante de baixo risco, observamos que há desajustes nos valores obtidos na medida da $\mathrm{AU}$. O interesse em avaliar as possíveis causas de variações das medidas da AU torna-se alvo do nosso interesse em pesquisa, sendo motivados a desenvolver um estudo que fundamente cientificamente as observações que temos feito. Reconhecendo que estas variações são influenciadas não apenas por características sociodemográficas, mas também por fatores de ordem metodológica, buscamos na literatura subsídios para identificar os fatores de variabilidade na obtenção das medidas de $\mathrm{AU}$.

Diante do apresentado, formulamos a hipótese de que a variabilidade intra-observador da medida da AU pode interferir consideravelmente na sensibilidade da curva de AU adotada pelo $\mathrm{MS}^{(18)}$, quando utilizada para rastrear desvios de crescimento fetal na gestação, e que um treinamento específico dos profissionais que realizam a medida de $\mathrm{AU}$ diminui o viés de aferição.

Dessa forma, este estudo teve como objetivo avaliar a efetividade de um curso de capacitação para enfermeiros e graduandos de Enfermagem na redução do viés de aferição na medida de altura uterina na assistência pré-natal. Além disso, também tivemos como meta: verificar a variabilidade intra e interobservador da medida da $\mathrm{AU}$ em gestantes; realizar um curso de capacitação em medida de AU pela técnica recomendada pelo Ministério da Saúde; verificar a variabilidade intra e interobservador da medida da AU em gestantes pós-intervenção; e comparar a variabilidade intra e inter-observador da medida da $\mathrm{AU}$ antes e após a intervenção (curso de capacitação).

\section{METODOLOGIA}

A pesquisa proposta teve um delineamento de estudo observacional, do tipo longitudinal e prospectivo. Foi composta a amostra por enfermeiros que atuam no serviço público de saúde de João Pessoa e que prestam assistência pré-natal, assim como graduandos do curso de Enfermagem da UFPB, selecionados por conveniência.

Para o cálculo do tamanho da amostra foram assumidas as seguintes condições:

a. a distribuição das médias amostrais da variável AU é gaussiana (normal);

b. o desvio-padrão da AU na população (s) não é conhecido, porém é supostamente constante em todas as idades gestacionais entre 13 e 39 semanas. Seu valor foi estimado (s) a partir dos dados da literatura. Optou-se pelo valor médio encontrado por Freire et al. ${ }^{(19)}, \mathrm{s}=1,1 \mathrm{~cm}$, por tratar-se de um estudo com a população paraibana;

c. a precisão de amostragem, ou seja, a amplitude máxima da diferença $(d)$ entre o valor da $A \cup(x)$ e o verdadeiro valor da $\mathrm{AU}(\mathrm{m})$, será $0,5 \mathrm{~cm}$.

O tamanho da amostra (n) foi calculado pela fórmula:

$$
n \geq\left(\frac{\left(z_{\alpha / 2}\right) \cdot S}{d}\right)^{2}
$$

Para um teste bicaudal ao nível de significância de 5\% $(\mathrm{a}=0,05), Z_{\alpha} / 2=1,96$, obteve-se então, $\mathrm{n} \geq 19$ indivíduos.

Para verificar a variabilidade intraobservador da medida da $\mathrm{AU}$, foi calculado o coeficiente de correlação intraclasse $(\mathrm{CCl})$ por ponto e por intervalo de $95 \%$ de confiança (IC95\%) entre as medidas, e as médias comparadas com o teste t pareado, com nível de significância de 5\%. Foi calculado também o Erro Técnico de Medida (ETM) intraobservador utilizando-se a fórmula: 


$$
\operatorname{ETM}(\text { absoluto })=\sqrt{\frac{\Sigma \mathrm{d}_{\mathrm{i}}^{2}}{2 \mathrm{n}}}
$$

Para verificar a variabilidade interobservadores da medida da $A U$, foi calculada a diferença entre as medidas de cada participante nas duas fases do estudo e o padrão-ouro adotado, que foi considerado como o valor da medida da $\mathrm{AU}$ de cada paciente realizada pelo investigador do estudo. $O$ valor da medida da AU obtida por cada participante foi comparado ao padrão-ouro para se calcular o viés de aferição. Em seguida, foram comparadas as médias dos desvios através do teste $t$ pareado, com nível de significância de 5\%. Foi utilizado o software livre Bioestat 5.0 para os cálculos estatísticos.

Foram levados em consideração os aspectos éticos da pesquisa envolvendo seres humanos recomendados pela Resolução $n^{\circ}$ 196/98 do Conselho Nacional de Saúde, principalmente no que diz respeito ao Consentimento Livre Esclarecido, para os indivíduos que realizaram as medidas da AU (enfermeiros e graduandos de Enfermagem) e para as gestantes que foram avaliadas para medição da AU fazerem parte desta pesquisa. Como o estudo foi exploratório e observacional, o resultado da medida da AU não modificou a conduta médica, que continuou a ser orientada pelos critérios clínicos e laboratoriais dos protocolos de conduta já definidos em cada Serviço assistencial.

A pesquisa foi cadastrada no SISNEP sob o registro $n^{\circ} 255145$ e foi aprovada pelo Comitê de Ética em Pesquisa da instituição.

A medida da $A U$, instrumento do estudo, foi obtida com o uso de uma fita inelástica sem escala (fita em branco). $\mathrm{O}$ examinador marcou o ponto que corresponde ao fundo uterino com uma caneta e entregou a fita à pesquisadora, que procedeu à leitura da medida da AU com uma escala milimetrada (precisão da medida $1 \mathrm{~mm}$ ). O padrão-ouro adotado foi a medida da $\mathrm{AU}$ realizada pela pesquisadora.

Cada um dos dois participantes do estudo realizou a medida da $\mathrm{AU}$ em dois momentos do estudo. Na $1^{\text {a }}$ fase do estudo (fase pré-intervenção), foi realizada a medida da AU de 20 pacientes selecionados pela pesquisadora com idade gestacional entre 13 e 39 semanas. Cada participante fez duas medições de cada uma das 20 pacientes, com duas fitas em branco distintas, sem saber previamente o resultado da medida anterior. Em seguida, foi ministrado um curso teórico-prático de capacitação em medida de altura com duração de 4 horas, em que foram desenvolvidas habilidades clínicas para a medição da AU pela técnica recomendada pelo Ministério da Saúde.

$\mathrm{Na} 2^{\mathrm{a}}$ fase do estudo (fase pós-intervenção), cada participante realizou a medida da $\mathrm{AU}$ das 20 pacientes de maneira semelhante ao procedimento da $1^{\text {a }}$ fase.

\section{RESULTADOS E DISCUSSÕES}

Na primeira fase do estudo, a variabilidade intraobservador, estimada pela média dos desvios entre as duas medidas de uma mesma paciente, foi 0,84 $\pm 0,62 \mathrm{~cm}$ (média \pm desvio-padrão). $\mathrm{O} \mathrm{CCl}$ por ponto e por intervalo de $95 \%$ de confiança (IC95\%) entre as medidas foi 0,989 (IC95\%: 0,980 - 0,994). O ETM absoluto foi $0,73 \mathrm{~cm}$ e o ETM relativo foi $2,25 \%$. Na segunda fase, a variabilidade intraobservador estimada pela média dos desvios entre as duas medidas de uma mesma paciente foi 0,58 $\pm 0,51 \mathrm{~cm}$ (média \pm desvio-padrão). $\mathrm{O} \mathrm{CCl}$ entre as medidas foi 0,994 (IC95\%: 0,988 - 0,997). O ETM absoluto foi 0,49 $\mathrm{cm}$ e o ETM relativo foi $1,51 \%$. O teste $t$ pareado mostrou que houve redução estatisticamente significante $(p<0,0001)$ na variabilidade intraobservador, o que tornou as medidas de $\mathrm{AU}$ menos variáveis, ou seja, mais precisas.

Para demonstrar a efetividade do curso de capacitação em melhorar a repetibilidade da medida da $\mathrm{AU}$, compararam-se as médias dos desvios entre duas medidas de uma mesma paciente antes e após a capacitação. Quando as médias dos desvios foram comparadas através do teste $t$ pareado, com nível de significância de 5\%, mostrou-se que houve diminuição estatisticamente significante nos desvios entre duas medidas, o que refletiu na diminuição do ETM entre a primeira e a segunda fase do estudo. Na Figura 1, estão representadas as distribuições dos desvios entre duas medidas da AU antes e depois do curso de capacitação. Comparando os gráficos box-plot, verificou-se redução tanto na média quanto na dispersão dos desvios.

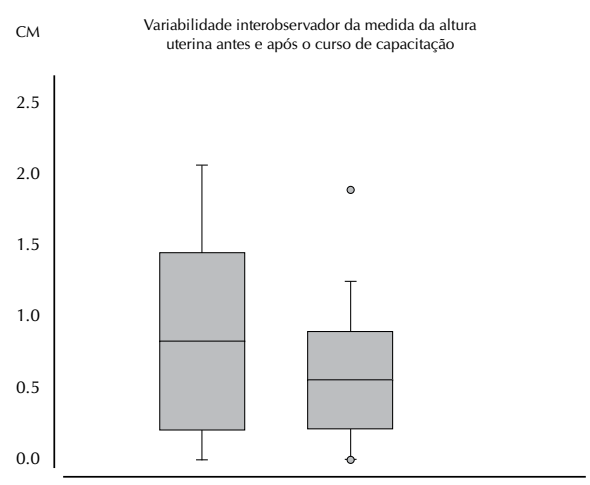

Figura 1 - Gráfico Box-plot dos desvios entre duas medidas da altura uterina realizadas antes e após o curso de capacitação na técnica de medição da altura uterina.

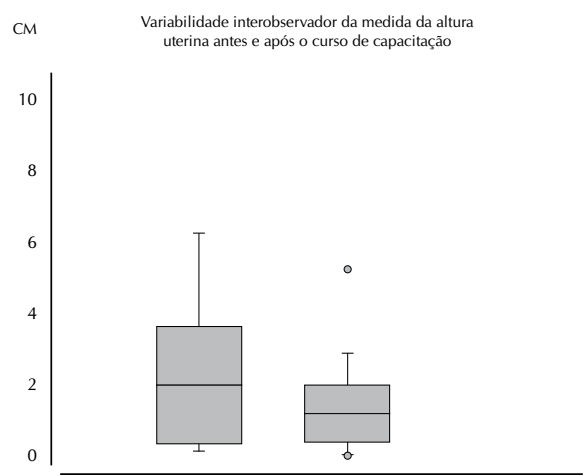

Figura 2 - Gráfico Box-plot dos desvios entre a média das duas medidas da altura uterina e o padrão-ouro (variabilidade interobservadores), realizadas antes e após o curso de capacitação na técnica de medição da altura uterina.

A fiabilidade da medida da AU foi estimada pela diferença entre a média das duas medidas de cada participante e 
o padrão-ouro. A precisão das medidas na primeira fase foi $2,01 \pm 1,66 \mathrm{~cm}$ (média \pm desvio-padrão) e na segunda fase foi $1,36 \pm 1,24 \mathrm{~cm}$. O teste $t$ pareado mostrou que houve redução estatisticamente significante $(p=0,0396)$ no viés de aferição, o que tornou as medidas de AU mais acuradas em comparação com o padrão-ouro adotado (Figura 2).

\section{CONCLUSÕES}

A capacitação de enfermeiros e graduandos de Enfermagem na técnica de medição da altura uterina reduz significativamente a variabilidade intraobservador e interobservadores e os erros técnicos de medida absoluto e relativo. Isto implica em melhor reprodutibilidade e maior fiabilidade das medidas da AU na prática clínica, o que poderá resultar em resultados clínicos mais homogêneos e em melhor sensibilidade e especificidade da medida da AU. Os resultados deste estudo apontam para a necessidade de investimentos em capacitação para os profissionais de saúde que realizam assistência pré-natal, tanto em nível dos cursos de graduação quanto em educação continuada para os já formados.

\section{REFERÊNCIAS}

1. Ministério da Saúde. Assistência pré-natal. 3. ed. Brasília: Centro de Documentação do Ministério da Saúde; 2000. p. 65.

2. Engstrom JL, Sittler CP. Fundal Height measurement: Techniques for measuring fundal height. J Nurse-Midwifery 1993;38(1 Pt 1):5-16.

3. Engstrom JL, McFarlin BL, Sampson MB. Fundal Height measurement: Accurracy of clinicians identification of the uterine fundus during pregnancy. J Nurse-Midwifery 1993;38(6 Pt 4):318-23.

4. Engstrom JL, Sittler CP. The effect of clinician bias on fundal height measurement. J Nurse-Midwifery 1994;39(Pt 5):130-41.

5. Pando R. Bases metológicas para evaluar el crescimiento fetal (I): Altura uterina por semana de gestacion. Rev Soc Bol Ginec Obst 1979;5(2):51-6.

6. Cunha SP, Ribeiro JU, Berezowsky AT, Duarte, G. Evolução da altura uterina e circunferência abdominal em gestantes normais. Rev Paul Med 1985;103(5):231-4.

7. Cherstani MAD, Santos IS, Cesar JA, Winckler LS, Gonçalves TS, Neumann NA. Assistência à gestação e ao parto: resultados de dois estudos transversais em áreas pobres das regiões Norte e Nordeste do Brasil. Cad Saúde Pública $2008 ; 24(7): 1609-18$.

8. Gonçalves CV, Costa JSD, Duarte G, Marcolin AC, Lim LCV, Garlet G, et al. Avaliação da freqüência de realização do exame físico das mamas, da Colpocitologia Cervical e da ultrassonografia obstétrica durante a assistência pré-natal. Uma inversão de valores. Rev Assoc Med Bras 2009;55(3):290-5.

9. Freire DMC, Paiva CSM, Coelho EAC, Cecatti JG. Curva da altura uterina por idade gestacional em gestantes de baixo risco. Rev Bras Ginecol Obstet 2006;28(1):3-9.
10. Pommier M, Escobedo F, Lowenberg E. Estudio de la altura uterina del fondo uterino para la detección del crecimiento intrauterino retardado. Ginecol Obstet Mex 1979;46(276):253-9.

11. Freitas $C B$. Evaluación de la altura uterina durante la gravidez. Rev Latinoam Perinatol 1986;6(4):128-32.

12. Jacobsen G. Prediction of fetal growth deviations by use of symphysis-fundus height and ultrasonic measurements. Int J Technol Assess Health Care 1992;(8 Suppl 1):152-9.

13. Ogunranti JO. Fundal height in normal pregnant Nigerian women: anthropometric gravidogram. Int J Gynecol Obstet 1990;33(4):299-305.

14. Margotto PR. Intrauterine growth curves: study of 4413 single live births of normal pregnancies. J Pediatr 1995; 71(1):11-21.

15. Hakansson A, Aberg A, Nyberg P, Schersten B. A new symphysis-fundus height growth chart based on a well defined female population with ultrasound-dated singleton pregnancies. Acta Obstet Gynecol Scand 1995;74(9):682-6.

16. Buhmann L, Elder WG, Hendricks B, Rahn K. A comparison of Caucasian and Southeast Asian Hmong uterine fundal height. Acta Obstet Gynecol Scand 1998;77(5):521-6.

17. Martinelli S, Bittar RE, Zugaib M. Proposta de nova curva de altura uterina para gestações entre a $20^{\mathrm{a}}$ e a $42^{\mathrm{a}}$ semana. Rev Bras Ginecol Obstet 2001;23(4)235-41.

18. Fescina RH, Quevedo C, Martell M, Nieto F, Schwarcz R. La medida de la altura uterina como método sencillo para predicir el crecimento fetal. Montevideo: Centro Latinoamericano de Perinatologia e Desarrollo Humano; 1983. p. 13.

19. Freire DMC. Curva de crescimento de altura uterina em gestantes atendidas na rede pública de João Pessoa-PB. João Pessoa. Dissertação [Mestrado]- Centro de Ciências da Saúde, Universidade Federal da Paraíba; 2002. p. 78. 